\title{
TRANSVERSAL DEFLECTION OF ELECTRONS MOVING IN PARALLEL WITH LINEARLY POLARIZED LASER BEAM AND ITS APPLICATION*
}

\author{
E.D. Gazazyan, K.A. Ispirian, M.K. Ispiryan, YerPhI, Yerevan, Armenia \\ D.K. Kalataryan, D.A. Zakaryan", YSU, Yerevan, Armenia
}

\section{Abstract}

The motion of electrons in linearly polarized laser beams in a finite length interaction region and then on a field free drift length is investigated. It is shown that in the interaction region the trajectories of the electrons are almost straight lines with very small oscillations. In the drift region the electrons acquire significant transversal deflection that allows to carry out the measurement of the length and longitudinal particle distribution of femtosecond bunches. The dependence of this deflection upon the electron energy, interaction region length, etc is studied. The principles of the construction of femtosecond oscilloscopes are discussed.

\section{INTRODUCTION}

At present when attosecond pulses are produced and even a proposal [1] for obtaining zeptosecond $\left(10^{-21} s\right)$ electromagnetic bursts has been published, there are methods for measurement of the characteristics of femtosecond (fs) laser pulses. However, only 30-100 fs electron bunches have been produced [2,3], and though some methods for obtaining [4] of femtosecond electron bunches have been proposed, there is no simple, time domain method [5] for their measurements.

Following [6] after considering the motion of electrons in a finite length interaction region with circularly polarized laser photons and then in a field free region in the works $[7,8]$ it has been proposed a time domain method for the measurement of the length and particle distribution of femtosecond electron bunches. In this work the same problems are considered for the case of linearly polarized plane wave (see Fig. 1).

\section{THE SCANNING OF THE ELECTRON BUNCH AND PRINCIPLES OF THE MEASUREMENTS}

For the fields of linearly polarized laser photons with frequency $\omega=c k$

$$
E_{y}=H_{z}=E_{0} \cos \left(\omega t-k x+\varphi_{0}\right) \text {, }
$$

solving the equation of motion of electrons in the interaction region with length $L_{\text {int }}$ between the mirrors $M 1$ and $M 2$ one finds the solutions in the form

\footnotetext{
*Work supported by ISTC grant A-372

\# zakaryan_davit@ysu.am
}

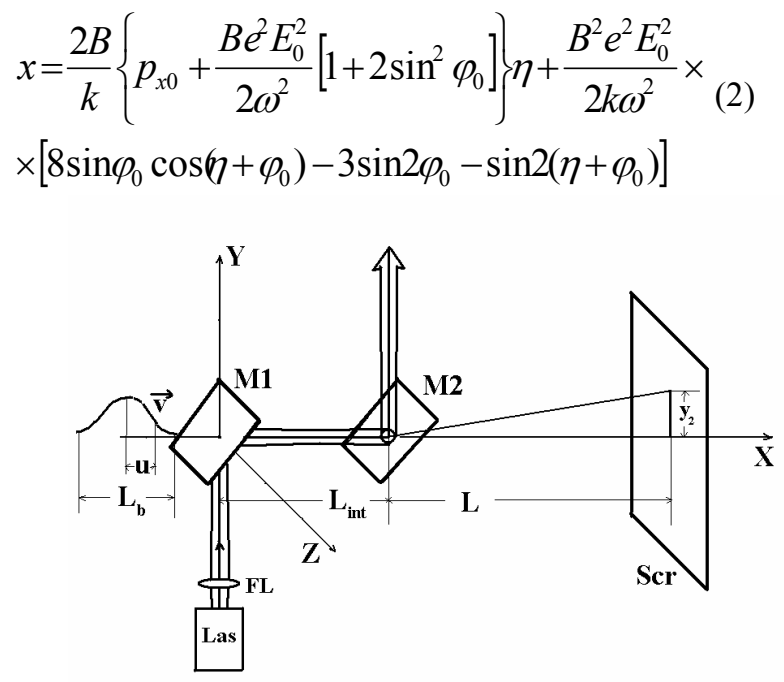

Figure 1: The experimental arrangement.

$$
\begin{gathered}
y=-\frac{2 B e E_{0} \sin \varphi_{0}}{k \omega} \eta-\frac{2 B e E_{0}}{k \omega}\left[\cos \left(\eta+\varphi_{0}\right)-\cos \varphi_{0}\right] \\
p_{x}=p_{x 0}+\frac{B e^{2} E_{0}^{2}}{\omega^{2}}\left[\sin \left(\eta+\varphi_{0}\right)-\sin \varphi_{0}\right]^{2} \\
p_{y}=\frac{e E_{0}}{\omega}\left[\sin \left(\eta+\varphi_{0}\right)-\sin \varphi_{0}\right]
\end{gathered}
$$

where $\varphi_{0}$ is the phase, $p_{x 0}$ is the initial momentum of the electrons, $\eta=\omega t-k x$ and the constant $B$ is equal

$$
B=\frac{\sqrt{m^{2} c^{2}+p_{x 0}^{2}}+p_{x 0}}{2 m^{2} c^{2}}=\frac{1}{2 m_{0} c}\left\{\gamma+\sqrt{\gamma^{2}-1}\right\}
$$

$$
\text { or when } \quad \gamma=\varepsilon / m c^{2}=\sqrt{1-(v / c)^{2}}>>1 \text {, }
$$

$B \approx \gamma / m c$. The coordinate of the electron $u$ (see

Fig.1) is connected with $\varphi_{0}$ by the relation $\varphi_{0}=u \omega / v=-2 \pi u / v T$ where $T=2 \pi / \omega$.

Fig.2 shows trajectories of $50 \mathrm{keV}$ electrons with $\varphi_{0}=0, \pi / 4, \pi / 2,3 \pi / 4, \pi, 5 \pi / 4,3 \pi / 2,7 \pi / 4$, in the interaction region for $C O_{2}$ laser field $(\lambda=10 \mu$ ) 
$E_{0}=2.10^{8} \mathrm{~V} / \mathrm{cm}$

, ( curves $1,2 \ldots 8$, respectively). The motion is 2 dimensional $(z=0)$ and in the frame where the electrons are in rest the trajectories have the form of "eight" in agreement with [9-11].

On the screen at a distance $L$ from the end of the interaction region

$y_{2}=y_{1}+\frac{p_{y 1}}{p_{x 1}} \cdot L \approx K L$

where $y_{1}$, the coordinate of electrons at the end of the interaction region due to drift and oscillations is small, and the so called bunch scanning factor is equal to

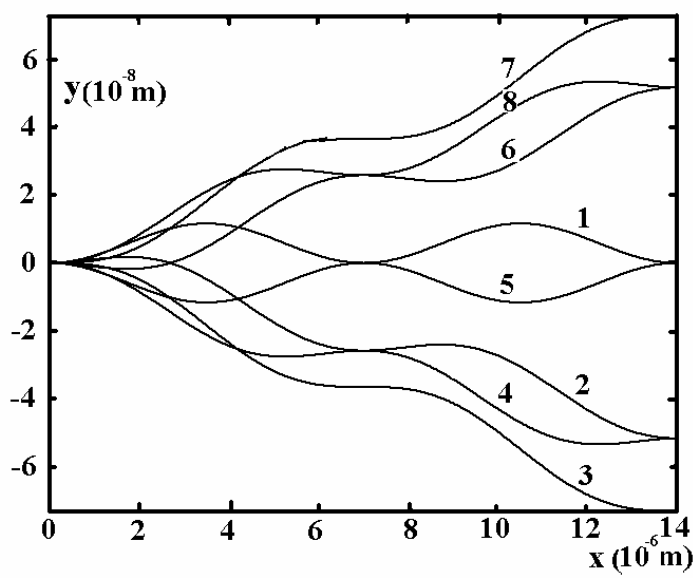

Figure 2: The trajectories in interaction region.

$$
K=\frac{p_{y 1}}{p_{x 1}}=\frac{A}{\sqrt{\gamma^{2}-1}+\frac{A^{2}}{2}\left[\gamma+\sqrt{\gamma^{2}-1}\right]},
$$

where

$$
A=\frac{e E_{0}}{m_{0} c \omega}\left[\sin \left(\eta_{1}+\varphi_{0}\right)-\sin \varphi_{0}\right],
$$

$\eta=\eta_{1}=\omega t_{1}-k L_{\text {int }}\left(t_{1}\right.$ is the time of electron fly through $L_{\text {int }}$ ).

The functions $y_{2}=y_{2}\left(\varphi_{0}\right)$ and $y_{2}=y_{2}(u)$ are non-linear, i.e. in contrast to the case of circular polarization in the case of linearly polarized photons the scanning of the electrons is non-linear. Nevertheless, measuring the initial and final points on the screen $y_{2}^{i}$ and $y_{2}^{f}$ one can measure the length of the electron bunch $T_{b}$ using (3). Measuring the distribution of the electron density on the screen $q_{2}\left(y_{2}\right)$ one can determine the initial distribution $q_{0}(u)$ of the electrons in the bunch using the following relation coming out from the charge conservation law

$$
q_{0}(u)=q_{2}\left(y_{2}\right)\left|\frac{d y_{2}(u)}{d u}\right| \text {. }
$$

\section{NUMERICAL RESULTS}

The below given results are obtained when some of the parameters have the values $\gamma=1.1, \mathrm{CO}_{2}$ laser field $E_{0}=2.10^{8} \mathrm{~V} / \mathrm{cm}, L_{\text {int }=}=5 \mathrm{~cm}, L=100 \mathrm{~cm}$. Fig 3 shows the dependence of $y_{2}=y_{2}(u)$.

Fig. 4 shows the dependence of the bunch scanning factor $K$ on $A$. As it is seen it is better to work at values of $A$ between the extrema of $K$

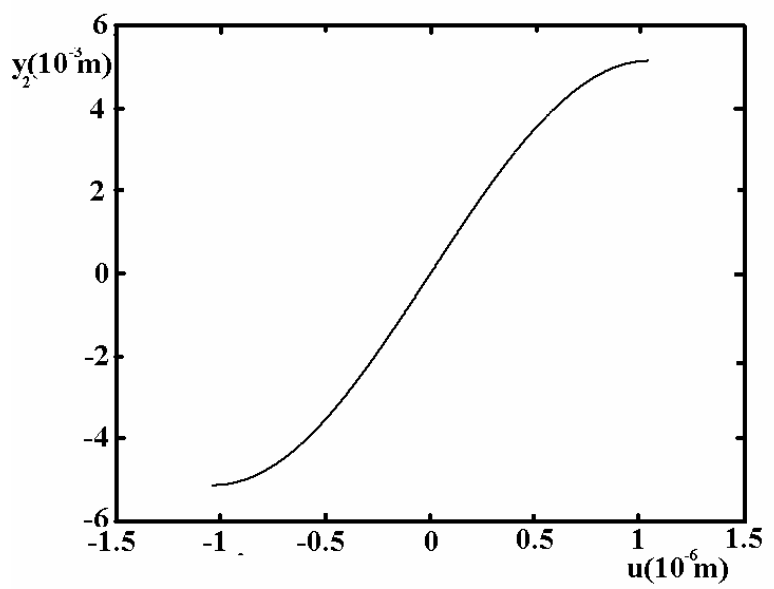

Figure 3: The dependence of $y_{2}=y_{2}(u)$.

taking place at $A= \pm \frac{\sqrt{2} \sqrt{\sqrt{\gamma^{2}-1}}}{\sqrt{\left(\gamma+\sqrt{\gamma^{2}-1}\right)}} \approx \pm 1$ for which one has $\frac{e E_{0}}{m c \omega} \approx 1$ or $E_{0} \approx 3.21 .10^{9} \mathrm{~V} / \mathrm{cm}$.

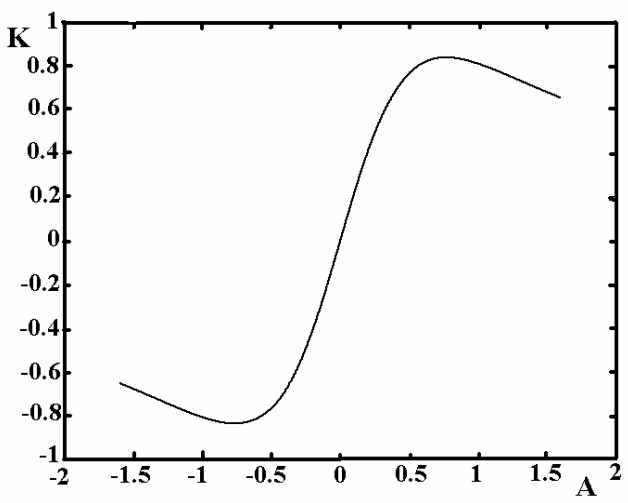

Figure 4: The dependence of $K$ upon $A$. 
Fig. 5 shows the dependence of the scanning factor $K$ upon the particle energy. It is seen that as it was expected the bunch image is larger for lower electron energies.

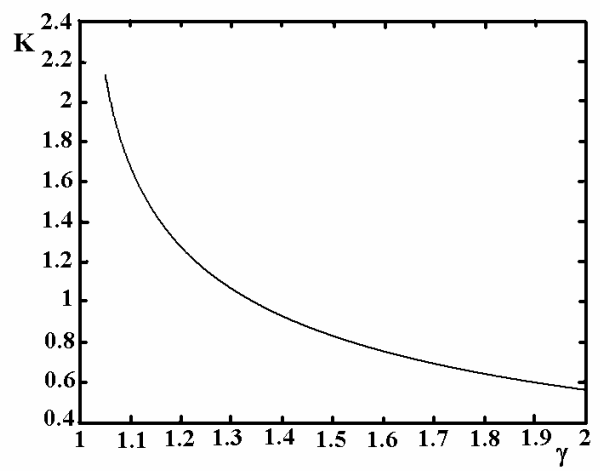

Figure 5: The dependence of $K$ upon $\gamma$.

For illustration, Fig. 6 shows how an electron bunch with length $L_{b}=2|u|=4 \mu m$ or $T_{b}=13.33$ fs and uniform particle distribution $q_{1}(u)=$ const (Fig.6a) gives an $2\left|y_{2}\right|=10 \mathrm{~mm}$ long image with none uniform particle distribution $q_{2}\left(y_{2}\right)$ on the screen of a $\mathrm{CO}_{2}$ oscilloscope with the above given parameters.

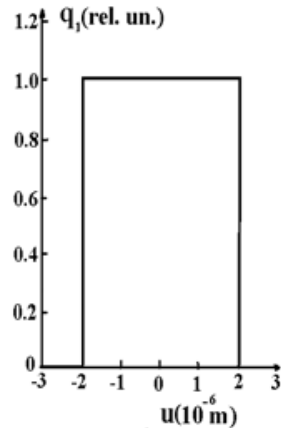

a)

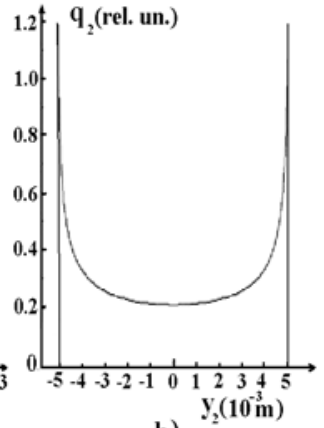

b)
Figure 6: The distributions of electrons in the bunch (a) and in the image on the screen (b).

\section{DISCUSSION AND CONCLUSION}

As it has been shown above the derived formulae allow to measure the length and the longitudinal particle density distribution of femtosecond electron bunches with the

help of linearly polarized laser photon beams and construct femtosecond laser oscilloscopes, though it is more difficult than in case of circular polarization $[7,8]$.
Using the derived relations and the dependences illustrated in figures one can choose optimal parameters of the device for the measurement of parameters of femtosecond electron bunches. These as well as other problems as the methods of detection of the scanned image and expected precision will be discussed in further publications.

\section{REFERENCES}

[1] A.E. Kaplan and P.L. Shkolnikov, Phys. Rev. Lett. 88 (2002) 074801.

[2] H. Lihn, P. Kung, C. Settakorn H. Wiedemann and D. Bochek, Phys. Rev. E53 (1996) 6413.

[3] V. Ayvazyan et al, Phys. Rev. Lett.88 (2002) 104802.

[4] P. Emma, K. Bane, M. Cornacchia, Z. Huang, G. Stupakov and D. Walz, Phys. Rev. Lett. 92 (2004) 074801

[5] G.A. Kraft, Proc. of 3-rd European Workshop on Beam Diagnostics and Instrumentation for Particle Accelerators, DIPAC, Frascati, Italy, 1997, p.48.

[6] K.A. Ispirian and M.K. Ispirian, Femtosecond Transversal Deflection of Electron Beams with the Help of Laser Beams and Its Possible Applications, ArXiv:hep-ex/0303044, 2003.

[7] E.D.Gazazian, K.A.Ispirian, M.K.Ispirian

D.K.Kalantaryan and D.A.Zakaryan, Femtosecond Scanning, Chopping and Length measurement of Electron Bunches by Laser Pulses and Principles of Femtosecond Oscilloscopes. To be published in the Proc. of NATO ARW «Advanced Photon Sources and Their Applications», Nor Hamberd, Armenia, August 29-September 2, 2004.

[8] E.D.Gazazian, K.A.Ispirian, E.M. Laziev, A.T. Margarian, D.K. Kalantaryan, Preliminary design of a femtosecond oscilloscope, To be published in the Proc. of PAC05.

[9] L.D. Landau and E.M. Lifshits, Teorya Polya, 5-th edition, Nauka, Moscow, 1967 (in Russian).

[10] E.S. Saranchik and G.T. Schappert, Phys. Rev. D1, 2738 (1970) 2738.

[11] B.M. Bolotovski and A.V. Serov, Uspekhi Fiz. Nauk, 173 (2003) 66. 